\title{
La comunicación como instrumento de expansión en la pequeña y mediana empresa española: el caso segoviano
}

\author{
Recibido: 15 de noviembre de 2010 \\ Aceptado: 27 de mayo de 2011 \\ Publicado: 31 de octubre de 2011
}

\author{
Begoña Gómez Nieto \\ mbgomez@uemc.es \\ Universidad Europa Miguel de Cervantes (España)
}

Resumen: El desarrollo de acciones de comunicación en la pequeña y mediana empresa (PYME) española es una asignatura todavía pendiente. El desconocimiento de las posibilidades que ofrece la comunicación para llegar a los públicos de una PYME, es una realidad manifestada, entre otros motivos, por la puesta en marcha de acciones llevadas a cabo por tradición.

Este artículo tiene como objetivo presentar una perspectiva del uso de la gestión de la comunicación en la pequeña y mediana empresa (PYME) española a través de un estudio empírico. Las conclusiones presentadas responden al estudio realizado con PYME exportadoras segovianas de diferentes sectores de actividad.

Palabras clave: Comunicación empresarial, PYME, pequeña y mediana empresa española, gestión de la comunicación, empresa exportadora.

Abstract: Implementation and development of communicative strategies is still incipient in the Spanish SME's market. Companies keep using old techniques instead of moving forward on innovative plans due to the lack of knowledge regarding the effectiveness of new programs.

This article offers a perspective on communication management in the mentioned framework based on an empirical research. The highlights have been given by foreign trade companies from Segovia (Spain) that operate in the broad range of activities covered by this study.

Keywords: Enterprise Communication, SME (Small and Medium Sized Companies), Spanish SME (Spanish small and Medium Sized Companies), Management of Communication, Exporting Company. 


\section{Introducción}

El desarrollo de acciones de comunicación ha estado ligado en las últimas décadas al ámbito de las grandes compañías. Así, la puesta en marcha de planes de comunicación no tendría cabida en la pequeña y mediana empresa. Este es el planteamiento que subyace en la mentalidad de muchos gerentes y responsables de PYMES cuando se hace referencia al término "comunicación". Todo gira en torno a los recursos y medios, y la limitación de estos condicionaría el uso y desarrollo de este tipo de acciones.

Lo cierto es que, en los últimos años, la pequeña y mediana empresa española es consciente de la importancia y de la necesidad de tener planes de comunicación perfectamente estructurados y desarrollados; partidas presupuestarias dedicadas a la puesta en marcha de acciones de comunicación; una identidad visual o un logo que impacte y cale en la mente del usuario; o una adecuada y eficaz estrategia para la gestión de su marca.

La situación, descrita a continuación, se corresponde con el período de duración del estudio empírico: desde mediados del 2006 hasta diciembre del 2007. En ese entonces, el tejido empresarial español estaba conformado principalmente por PYMES, las cuales integraban el $99.87 \%$ de las empresas registradas.

Si analizamos la previsión de crecimiento de las PYMES españolas respecto a Europa (los siete países representativos: Alemania, Bélgica, Francia, España, Italia, Portugal y Reino Unido), según los resultados del barómetro Eurofactor 2007 de Confianza Empresarial $^{1}$, sitúa a las PYMES españolas entre las más optimistas en aspectos como el volumen de negocio, la previsión de crecimiento, la rentabilidad, el entorno económico y la contratación. Los resultados obtenidos mostraron perspectivas muy diferentes para cada uno de los países.

Respecto a la previsión de crecimiento, España se situaba a la cabeza de los países de la Unión Europea con un 62\%, por delante de países como Reino Unido o Bélgica. En cuanto a volumen de negocio, se colocaba precisamente por detrás de estos dos países (con un $50 \%$ ). A pesar de que las PYMES españolas no son las que más confianza tenían en su entorno económico sí fueron las más seguras de su entorno. Son los propios empresarios españoles (63\%) los que consideran más fácil contratar a nuevo personal, debido a la importante oferta laboral que supone la inmigración.

A la vista de este párrafo introductorio nos damos cuenta del peso que tienen realmente las PYMES en el conjunto de la economía de nuestro país y nos cuestionamos si la comunicación es utilizada como un elemento que les ayuda a crecer en el panorama internacional en el que se mueven, siendo este cada vez más competitivo.

El objetivo principal de este artículo, por tanto, es presentar el panorama actual de la gestión de la comunicación en la PYME española a través de un estudio empírico realizado con PYMES exportadoras segovianas de diferentes sectores de actividad. Los resultados son extrapolables al resto de PYMES españolas de núcleos poblacionales similares al

1.- Este estudio, realizado por quinto año consecutivo, analiza pequeñas y medianas empresas de 6 a 500 empleados de los siete países europeos representativos mencionados. 
objeto de estudio. Por lo tanto, se averiguan las acciones de comunicación utilizadas por las PYMES segovianas y la justificación de dichas acciones. También constituye un punto de partida para realizar estudios similares desde diferentes ángulos ante un fenómeno de estudio escasamente analizado y no por ello menos importante: la comunicación en el colectivo de la PYME española.

Aclaramos que el estudio presenta una visión retrospectiva del panorama comunicativo y empresarial, puesto que los datos utilizados en el estudio corresponden a 2008, aunque las acciones comunicativas siguen perdurando en la actualidad, no así el panorama y la situación económica de las PYMES analizadas, sobre todo tras la crisis financiera que ha afectado a España en los últimos años.

\section{El diseño de la muestra}

El origen de las empresas segovianas analizadas es de diversa índole: algunas de ellas son una herencia familiar que hijos y nietos han sabido aprovechar, adaptándose a las nuevas condiciones del mercado; otras son iniciativa propia de personas con formación y visión de negocio para comenzar la actividad en el mercado local y paulatinamente se han ido expandiendo al ámbito regional, nacional e internacional.

El estudio empírico se realizó sobre el censo de PYMES segovianas, proporcionado por la Cámara de Comercio de Segovia, con el objetivo de extraer conclusiones que aporten claridad sobre el uso de la comunicación en este contexto empresarial.

Desde 1993, con la aplicación de la Ley de Protección de Datos, no existen bases de datos públicas de empresas exportadoras, ya que la Agencia de Aduanas dejó de proporcionar esta información. Existe, en la actualidad, una base de datos en el Consejo Superior de Cámaras y la Agencia de Aduanas, a donde acudimos (http://www.camaras.org/) para encontrar datos de las empresas exportadoras de España.

Las empresas exportadoras no tienen obligación de registrarse en ninguna base de datos, aunque normalmente acuden a la Cámara de Comercio local para pedir certificados que necesitan en su actividad exportadora. La Cámara de Comercio de Segovia confecciona, por iniciativa propia, un listado de las empresas a las que emiten dichos certificados y, además, poseen un listado de empresas de las que saben (debido a las gestiones que hacen con ellas) cuáles exportan, exportaban o están interesadas en la actividad exportadora.

Esto no significa que en ese listado estén todas las empresas que realmente exportan. Por lo tanto, partiendo de ese amplio listado, contactamos con las propias PYMES (mediante llamadas telefónicas o correo electrónico), preguntándoles si en ese momento exportaban y si estaban dispuestas a colaborar en la investigación. Así obtuvimos el listado definitivo de empresas a las que aplicamos nuestro cuestionario.

Las empresas de nuestro estudio debían cumplir unos criterios de inclusión para participar en el estudio: 
- Ser pequeña y mediana empresa conforme a la definición previa establecida.

- Ser PYME exportadora, entendiendo la actividad exportadora como una actividad desarrollada de modo regular y no puntual.

- Estar dispuesta a participar en la investigación.

Como datos primarios, utilizamos las respuestas obtenidas en el cuestionario que haríamos llegar a las PYMES para que lo rellenaran a través del medio elegido por ellas (correo electrónico, fax, correo ordinario, entrega personal). Finalmente, fueron 33 empresas las que decidieron colaborar en el estudio. El nivel de participación de las empresas que cumplían los requisitos fue de un $80.48 \%$.

Se utilizó una técnica cuantitativa de diseño no experimental, como es el cuestionario. Dicho instrumento fue proporcionado a los gerentes o responsables de las PYMES segovianas para ser completado por ellos.

\section{El proceso metodológico y sus fases}

Las fases del proceso metodológico fueron las siguientes:

- Diseño del cuestionario. Teniendo en cuenta los objetivos a conseguir en la investigación, se estructuró un cuestionario constituido en su mayoría por preguntas cerradas y semiabiertas, basadas en tres núcleos: el proceso de internacionalización, la gestión de la comunicación en la PYME y las tendencias o previsiones de futuro en materia de comunicación por parte de las PYMES analizadas. Estas tres partes aparecen claramente diferenciadas en el cuestionario, separando los bloques de preguntas de cada una de ellas.

- Selección de aquellas empresas que cumplen los requisitos establecidos a priori. Del listado inicial suministrado por la Cámara de Comercio de Segovia fue necesario realizar varios filtros hasta conseguir el listado definitivo de empresas colaboradoras. Los gerentes o responsables de las PYMES que rellenaron el cuestionario pertenecían a empresas que cumplían los criterios de inclusión fijados de antemano: que las empresas fueran PYMES, que se tratase de PYMES exportadoras, que además desarrollasen la actividad de un modo regular y que aceptaran participar en la investigación.

- Contacto con los gerentes y/o responsables de PYMES. La fase de contacto con los gerentes de las PYMES exportadoras segovianas fue ardua y duradera en el tiempo, puesto que fueron necesarios una media de 3-4 contactos, ya que hablamos de personas con poca disponibilidad y que suelen viajar bastante. Se utilizaron diferentes medios de contacto: correo electrónico, teléfono fijo de la empresa y móviles profesionales y personales de los gerentes facilitados por la propia empresa.

- Entrega y recepción de cuestionarios. A través de diferentes medios (Internet, fax, correo postal y visita personal a la empresa). Se respetó el medio de entrega del cuestionario elegido por la empresa en cuestión, atendiendo a sus preferencias. En esta fase, el medio 
utilizado nos proporciona una visión de los recursos tecnológicos de los que dispone y utiliza la empresa.

- Análisis de resultados. Una vez recepcionadas las encuestas, se procedió a su análisis y la consiguiente presentación de resultados. El objetivo es estudiar, en detalle, la información aportada por el encuestado en base a los tres núcleos que presentamos en el cuestionario para interrelacionar las variables de estudio: a) el proceso de internacionalización, puesto que en este bloque de preguntas se plasma todas las cuestiones relacionadas con los orígenes de la empresa, productos que comercializa, cómo es su actividad exportadora; b) la gestión de la comunicación. En este grupo de preguntas nos interesa averiguar cómo gestionan las PYMES su labor de comunicación, qué acciones realizan, periodicidad, recursos, profesionales con los que trabajan, etc.; c) las tendencias de futuro de las PYMES exportadoras segovianas, puesto que también se quiere analizar las previsiones de futuro que tienen las PYMES segovianas en materia de comunicación: determinar las perspectivas que estas tienen y sus planes a corto y a medio plazo en este campo.

En este artículo consideramos oportuno presentar los resultados más relevantes correspondientes al segundo núcleo del cuestionario: la gestión de la comunicación.

\section{Resultados}

\subsection{Acciones de comunicación que realizan las PYMES analizadas}

La asistencia ferial es la acción comunicativa más desarrollada por las empresas segovianas encuestadas en su camino hacia la internacionalización. El 70\% de las PYMES analizadas asiste a ferias. De este marge, el $52.17 \%$ asiste tanto a ferias nacionales como internacionales; un $26.08 \%$ acude sólo a las nacionales y un $21.73 \%$ asiste a ferias sólo de carácter internacional. Las ferias nacionales mencionadas por las PYMES encuestadas son la feria Agroalimentaria de Barcelona, Madrid (Alimentación) y Riberexpo (vinos).

Entre las ferias internacionales mencionadas por los encuestados destacan la de Sial en Francia, Lisboa, Anuga y Frankfurt (Alemania), todas del sector agroalimentario. El $78.79 \%$ de las PYMES analizadas dispone de página web, aunque no siempre se saque el máximo rendimiento de esta.

Esta característica coincide con el resultado de un estudio realizado con empresas extremeñas por la doctora Carrillo y sus colaboradoras donde queda patente, al igual que en el caso segoviano, la infrautilización de la página electrónica. No existen posibilidades de comercio electrónico: la utilización de este soporte se realiza para dar mayor difusión a los negocios que se crean y gestionan fuera de la red (Carrillo, Castillo y Gómez, 2005:130).

En cuanto a otras acciones de comunicación, el 75.76\% utiliza catálogos de productos. El catálogo constituye la herramienta fundamental en la presentación de productos a clientes de manera directa, es decir, one to one. Por lo tanto, la comunicación de producto ocupa un papel muy importante dentro de las empresas analizadas, más que la comunicación empresarial propiamente dicha; es decir la presentación de la empresa en un sentido global. 
Un $60.61 \%$ emplea revistas del sector; esto es, especializadas. El 51.52\% emplea folletos de productos. El $27.27 \%$ utiliza prensa nacional. El 15.15\% emplea el medio radio en ámbito nacional, a través de cuñas, patrocinio o colaboraciones esporádicas en programas. Sólo un $9.09 \%$ utiliza la televisión en el ámbito nacional debido a su alto coste y a su dudosa eficacia. Debemos matizar que, en el camino hacia la expansión de productos fuera de las fronteras nacionales, las acciones comunicativas deben desarrollarse en el ámbito internacional. El empleo de prensa, radio y televisión centrados en el entorno nacional no contribuyen a asentar los productos de la empresa en los mercados extranjeros.

En la figura 1, se presentan las acciones de comunicación desarrolladas por las empresas en el que destacan los porcentajes altos en el uso de catálogos, páginas web y asistencia ferial, que contrastan con el escaso uso de la televisión y la radio de ámbito nacional.

Figura 1: Acciones de comunicación desarrolladas por las PYMES.

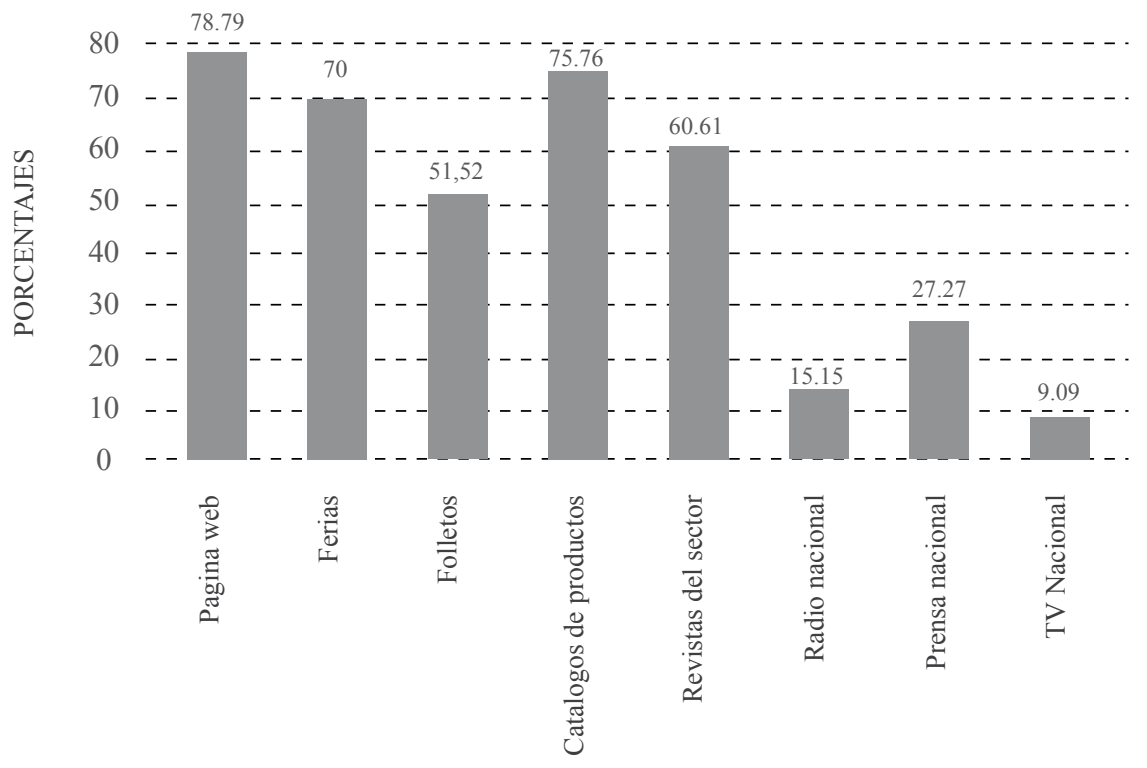

ACCIONES DE COMUNICACIÓN

Fuente: Elaboración propia.

En cuanto a la participación en ferias de las empresas analizadas, destacamos lo siguiente:

- Un $81.82 \%$ tiene un stand compartido, respondiendo a la necesidad de ahorro de costes.

- Otro $78.79 \%$ utiliza el stand subvencionado por organismos e instituciones, aprovechando las ayudas ofrecidas por las entidades a las PYMES exportadoras. 
- El 18.18\% cuenta con stand propio. Se trata de empresas con experiencia en la participación de ferias y con una capacidad económica solvente que permite a la empresa soportar los costes derivados del diseño, montaje y puesta en marcha del stand.

- Sólo un 3.03\% dispone de las tres modalidades de stand: propio, compartido y subvencionado.

Respecto al personal que asiste a las ferias:

- En el $69.70 \%$ de los casos analizados, se acude a las ferias con personal externo contratado (azafatas o personal experto).

- En el $30.30 \%$ de los casos, se asiste a las ferias con personal propio de la empresa, generalmente el gerente o responsable de la PYME (entre 1 y 2 empleados). Ante este porcentaje, deducimos que no se explota adecuadamente el hecho de que el personal propio de las empresas asista a las ferias, siendo las personas más aptas para estar en ellas, puesto que conocen sus productos y la empresa a la perfección, pudiendo informar y captar mejor a nuevos clientes y mantener los existentes.

Los principales objetivos que tienen las empresas a la hora de asistir a las ferias son los siguientes:

- El $65.21 \%$ de los que asisten a ferias pretende desarrollar el contacto y la captación de potenciales clientes. Y también el mantenimiento de clientes ya existentes. La importancia del contacto con el cliente para este tipo de empresas es crucial, ya que es la base del beneficio económico por las ventas de los productos.

- Un $52.17 \%$ busca presentar los productos y dar a conocer los mismos.

- Un 26.08\% pretende dar a conocer la compañía.

- Un $17.39 \%$ intenta contactarse con el sector de forma directa y conocer las tendencias del mercado.

- El 13.04\% sigue bjetivos comerciales y cerrar ventas.

- Fortalecer y afianzar la imagen corporativa es señalado por el 13.04\% de las PYMES asistentes a las ferias. Este bajo porcentaje indica que la comunicación empresarial (corporativa) queda por debajo, en nivel de importancia, respecto a la comunicación de producto.

- Contacto con distribuidores potenciales (8.69\%).

- Un $4.34 \%$ desea analizar su producto comparándolo con la competencia.

- Un $4.34 \%$ considera que es necesario asistir a ferias para tener una presencia en el mercado.

- Otro 4.34\% apunta como objetivo la promoción de la imagen y el prestigio.

- Se señala también la captación de oportunidades comerciales (4.34\% de los asistentes). 
- Conseguir contactos en materia de innovación (4.34\%).

- Intercambio de información con otras empresas del sector (4.34\%).

En cuanto a la eficacia de la asistencia ferial:

- El $72.72 \%$ de los encuestados considera que es eficaz, aunque señala que los resultados se obtienen a largo plazo.

- Un $15.15 \%$ apunta la ineficacia debido a la masificación y al alto coste que implica la asistencia ferial.

- Un $12.12 \%$ de los encuestados no sabe o no contestó la pregunta.

\subsection{Periodicidad de las acciones de comunicación}

En lo relativo a la periodicidad o frecuencia del desarrollo de las acciones de comunicación, señalamos lo siguiente:

- E1 70\% de las PYMES encuestadas realiza acciones de comunicación.

- Un 36.36\% desarrollan anualmente acciones de comunicación.

- El 21.21\% de los encuestados no responde a la cuestión.

- Otro $18.18 \%$ señala que realizan dichas operaciones de forma trimestral.

- Un $12.12 \%$ lo desarrolla con carácter semestral.

- Un $6.06 \%$ de los encuestados mantiene periodicidad mensual.

- Un 3.03\% tiene una periodicidad bienal.

La frecuencia varía dependiendo del tipo de acción comunicativa: si realizan folletos (mensual) si se trata de prensa y revistas especializadas (trimestral) y si acuden a ferias lo hacen anualmente o cada dos años. A las ferias se asiste con periodicidad anual o bienal, dependiendo del carácter de la feria. Las internacionales suelen ser bienales, las de carácter nacional tienen lugar una vez al año.

\subsection{El papel de la comunicación en el proceso de internacionalización}

Respecto al papel de la comunicación en el proceso de internacionalización de la empresa, el $72.73 \%$ de las PYMES analizadas considera que es positivo por las siguientes razones (extraemos las frases textuales de los encuestados):

- "La comunicación es totalmente necesaria para dar a conocer un producto, su calidad y dar a conocer la imagen de la empresa".

- "Aporta clientes y ayuda a que el consumidor comprenda la diferencia de precios, porque éste busca referencias a través de la comunicación". 
- "Ayuda a la empresa a conocer nuevos mercados".

- "Ayuda a la empresa a conocer las tendencias del mercado y el sector en el que se mueve".

- "Mantiene viva a la empresa en el mercado y aporta imagen de seriedad a la misma".

• "Es una forma de establecer relación con potenciales clientes y mantener los ya existentes".

- "Ayuda a contactar directamente con proveedores y clientes".

- "Mediante la comunicación se hace la presentación de avances y estudios realizados".

- "Es necesario desarrollar una buena comunicación, teniendo en cuenta aspectos como el rigor de la información, contar con personas cualificadas en idiomas sobre todo y dar prioridad en el servicio atención al cliente".

Un $15.15 \%$ manifiesta su indiferencia y un $12.12 \%$ no sabe/no contesta.

\subsection{El plan de comunicación}

En lo referente a los planes de comunicación a nivel nacional (de corto/mediano plazo), el $45.45 \%$ de las entidades encuestadas no los tiene; un 33.33\% sí dispone de estos, aunque en la mayoría de los casos aparecen poco definidos. Un $21.21 \%$ no sabe/no contesta. En lo que respecta a la posesión de planes de comunicación orientadas a la exportación, el $56.52 \%$ señala que no los tiene; un $26.09 \%$ dispone de estos y un $17.39 \%$ no sabe/no contesta.

Las PYMES que cuentan con un plan de comunicación para la exportación poseen también uno diseñado para el ámbito nacional o, al menos, esbozado. Consideran la comunicación de forma global y no como acciones puntuales e independientes. Asimismo, las empresas que apuestan por la comunicación han logrado, en los últimos cinco años, tener una posición sólida en los mercados internacionales y han aumentado su nivel de facturación. Bien es cierto que a la comunicación no se le puede atribuir enteramente el éxito o el fracaso del desarrollo de la actividad de una empresa, ya que son múltiples factores los que intervienen. Sin embargo, la comunicación se yergue como un elemento importante, que contribuye positivamente a la consecución de los objetivos.

De estos resultados se deduce que la mayoría de las PYMES segovianas no dispone de un plan de comunicación debidamente elaborado. Cuando se solicitó a los encuestados describir sus respectivos planes de comunicación, apenas aportaron datos específicos, aunque hicieron incidencia en el plan de ferias ("Realizamos un plan de ferias con calendario y presupuesto cerrado de un año para otro, tanto nacionales como internacionales"). También se hace referencia al desarrollo de eventos, los cuales recibirían un "presupuesto conjunto cada año para realizar actuaciones tanto en radio, televisión, revistas especializadas, asistencia a ferias, entre otros", pero no se profundiza más ("Participamos en eventos y asociaciones, y visitas a países").

Del mismo modo, queda reflejado en las respuestas que la elaboración de los planes de comunicación (sobre todo las enfocadas en la planificación de la asistencia ferial) se llevan a cabo por costumbre y hábito. 


\subsection{Existencia de personal cualificado en materia de comunicación}

El 51.51\% de las empresas encuestadas no dispone de personal cualificado, mientras que el $42.42 \%$ cuenta con personal cualificado propio; y un $6.06 \%$ no sabe o no contesta a la cuestión. El plan de comunicación tiende a ser ejecutado por el personal cualificado de la empresa, aunque para el desarrollo de las diversas acciones de comunicación (dependiendo del tipo de acción) se cuenta con la participación de personal externo.

Para desarrollar sus acciones de comunicación, el 45.45\% de las PYMES no cuenta con colaboraciones externas, mientras que un $42.42 \%$ acude a los servicios externos de profesionales. Un $12.12 \%$ de los encuestados no sabe/no contesta.

Dentro de las colaboraciones externas, los servicios más demandados por las PYMES analizadas son los desarrollados por:

- Agencias de publicidad, profesionales independientes, traductores.

- Personal de los medios de comunicación (prensa, radio, televisión).

- Empresas de comunicación especializadas en la construcción de marcas e identidad corporativa. Las agencias de publicidad contratadas suelen ser locales, pero también de provincias cercanas (Madrid, Valladolid).

- Diseñadores.

- Colaboración puntual de imprentas para la realización de catálogos.

\subsection{La evaluación de la eficacia de las acciones de comunicación}

El $72.72 \%$ de los encuestados no elabora dicha operación, frente a un $27.27 \%$ que sí lo hace. El primer porcentaje es un dato significativo, ya que revela el desinterés hacia el proceso de evaluación de las acciones de comunicación, ignorando si estas fueron eficaces o no. En todo caso, las respuestas que se obtuvieron son las siguientes:

- Es difícil evaluar la eficacia de la asistencia ferial (es la acción de comunicación más utilizada) porque son resultados visibles a largo plazo (un año y medio o dos años) pero aún así es positivo.

- La eficacia es mayor cuanto más próxima sea la comunicación con el consumidor. Se trata de empresas pequeñas, por lo que la comunicación se centra más en acciones promocionales, donde el contacto con el consumidor es directo o realizado a través de asociaciones en las que están representados varios fabricantes. El asociacionismo, a todos los niveles, es útil y necesario para la pequeña y mediana empresa.

- "Se estudia la eficacia periódicamente según costes y evoluciones".

- Las empresas se dirigen a los clientes para averiguar si tienen un buen concepto del producto, si recuerdan la información y si esta es clara, pero sin profundizarse más. 
- Una empresa menciona que realizan una encuesta anual a clientes y llevan a cabo la recogida y clasificación de reclamaciones.

- "Se debe valorar con las ventas". Este comentario asienta la idea de la asociación "acción de comunicación = rentabilidad (a corto plazo)", lo cual confirma que la inversión en comunicación, por parte de las PYMES segovianas, es limitada por esta razón y por los pocos recursos económicos a la que se ve sometida en su quehacer cotidiano. Esta idea coincide con el estudio similar ya realizado a PYMES extremeñas (Carrillo, Castillo y Gómez, 2005: 132).

En el 18.18\% de los casos existe contradicción en las respuestas relacionadas con la comunicación, originada básicamente por cierta confusión o ambigüedad sobre conceptos relacionados con este término, por las siguientes razones:

- Se identifica "comunicación" con "tecnología". Por ejemplo, cuando se pregunta por los aspectos favorables que aporta a la empresa un buen desarrollo de la comunicación, se responde que es positivo porque "proporciona información operativa online en red privada, investigación de nuevos procesos, captación de datos macroeconómicos/evolución de mercados"; es decir, se hace referencia meramente a cuestiones tecnológicas.

- Se identifica "comunicación" con "información", apuntándose que es positivo el papel del primero en el proceso de internacionalización de la empresa porque "con la comunicación se aprende de mercados, se conoce nuevos mercados y a sus potenciales clientes", sobre todo cuando esta tiene múltiples objetivos distintos dependiendo de lo que le interese a la empresa.

- Al preguntarse si la empresa dispone de personal cualificado en materia de comunicación, los encuestados detallaron el perfil del personal implicado: vendedores, agentes comerciales en otros países y un ingeniero; es decir, se menciona el "equipo técnico cualificado" para el desarrollo de la actividad genérica de la empresa, pero no se trata de personal cualificado para desarrollar tareas específicas de comunicación.

- Se pregunta también si la empresa cuenta con un plan de comunicación de cara a la exportación. Cuando la respuesta es afirmativa, se pide a los encuestados que describan dicho plan, pero esta respuesta queda en blanco, de lo que se podría deducir que no está claro qué entienden en la empresa como "plan de comunicación".

La comunicación empresarial no es información que genera la empresa: la información es parte de la comunicación, pero no se reduce a ella. Mientras muchas empresas conceden un valor creciente a la comunicación, considerándola un factor diferencial que repercute en su imagen, en su productividad y en la calidad de vida laboral, en otras sigue predominando la "política de oídos sordos", de puertas cerradas, y sigue cometiéndose el error de identificar “comunicación” con "información”. La información es sólo una parte de ese proceso y por sí mismo no produce comunicación.

Según Carrillo, Castillo y Gómez (2005: 17), la comunicación empresarial es la forma en que una empresa se relaciona con sus públicos de interés internos (trabajadores principalmente) y externos (clientes, proveedores) a través de los productos que fabrica y 
de la información que difunde sobre ella misma (identidad).

Otra cuestión que se planteó es la explotación de los recursos de comunicación dentro de las empresas. Los resultados han sido los siguientes:

- El 42.42\% opina que las empresas explotan poco (o regular) los recursos de comunicación.

- El 24.24\% de los encuestados no sabe/no contesta, debido al desconocimiento sobre el tema de los recursos y acciones de comunicación. Si no lo conocen, no saben si lo están explotando o no. Así, la falta de evaluación de la eficacia (respecto al desarrollo de las acciones de comunicación) está relacionada con este desconocimiento.

- Un 21.21\% considera que la explotación es "suficiente". Los recursos de comunicación se utilizan acorde con las necesidades puntuales que van surgiendo en la empresa, pero sin grandes pretensiones.

- Sólo un $12.12 \%$ apunta que se explotan "bastante".

Ante estos resultados (figura 2), se percibe una actitud de conformismo ante los recursos de comunicación empleados (hasta el momento) en la empresa y las pocas ganas de innovar. Esta postura conformista (que se traduce en expresiones como: "Vamos poco a poco y por ahora nos da resultado") coincide con conclusiones de estudios similares.

Figura 2: Explotación de los recursos de comunicación.

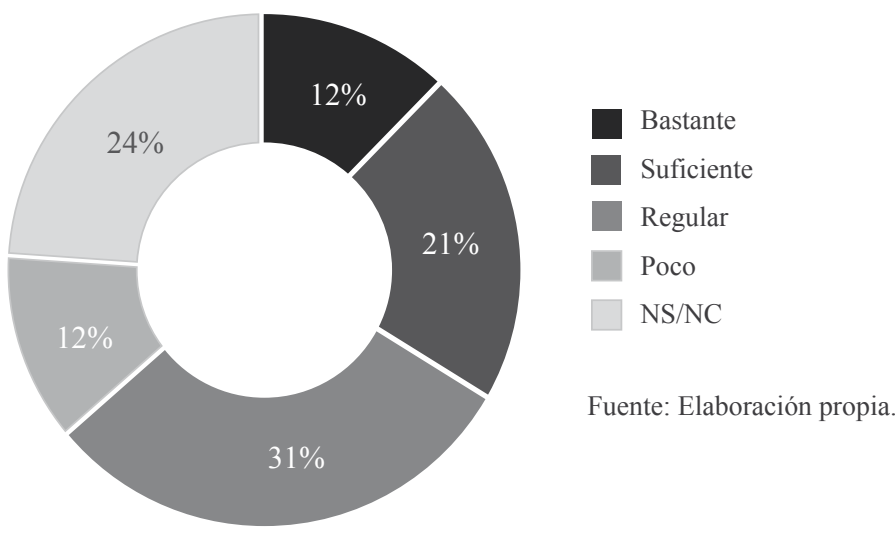

\subsection{La relación PYMES - agencias de comunicación}

- En cuanto a la opinión sobre la relación de la empresa con las agencias de comunicación (figura 3) destacamos los siguientes resultados:

- El 33.33\% opina que la relación a la hora de trabajar con ellos (y como receptores de sus servicios) es "buena".

- Otro 33.33\% opina que la relación es "normal”. 
- El 24.24\% de los encuestados no sabe/no contesta.

- Un $6.06 \%$ afirma que la relación con las empresas especialistas en comunicación es "mala".

- Sólo un 3.03\% señala que la relación es "muy buena".

Figura 3: Relación PYMES segovianas - agencias de comunicación.

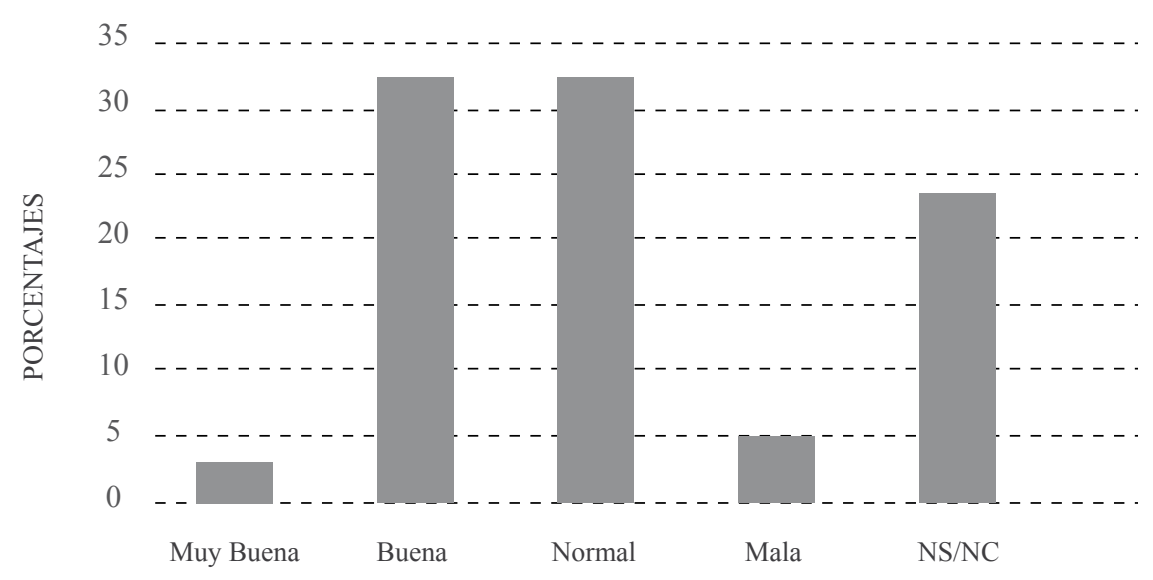

RELACIÓN EMPRESA SEGOVIANA - AGENCIAS COMUNICACIÓN

Fuente: Elaboración propia.

De las respuestas obtenidas se deduce que la relación con las agencias de comunicación es escasa. Esta se basa, fundamentalmente, en colaboraciones esporádicas y muy puntuales. Los servicios prestados son suficientes y el nivel de exigencia a los profesionales (para desarrollar acciones eficaces de comunicación) no es alto.

Cabe acotar que un $42.42 \%$ acude a los servicios de expertos, de los cuales sólo el 3.03\% experimenta una relación "muy buena", lo que significa que los servicios prestados por los profesionales no son tan satisfactorios. De esta manera, se deduce que los encuestados ponen en duda la profesionalidad de las agencias de comunicación. En la mayoría de los casos no estamos ante "expertos": las agencias no entenderían las demandas de las PYMES, mucho menos que deben proporcionar soluciones eficaces, adaptadas a los presupuestos (bastantes limitados) de las PYMES.

En definitiva, se constata la aparente carencia de las PYMES en el ámbito de las acciones de comunicación, con la finalidad de expandir sus productos fuera de las fronteras nacionales. 


\section{Conclusiones}

Las respuestas aportadas por las PYMES indican que el epígrafe "acciones de comunicación" engloba una variedad amplia de términos y acciones que no son precisamente acciones de comunicación. Las respuestas dadas por los responsables y gerentes convierten la comunicación (y sus diversas ramificaciones) en una especie de "cajón de sastre", en el que cabe todo.

En términos generales, se confunde "comunicación” con "información”, "tecnología", "atención al cliente", "formación de personal cualificado en comercio exterior", "en idiomas", entre otros. En suma, no definen con precisión las acciones de comunicación propiamente dichas. Este planteamiento (junto con las respuestas ambiguas en esta materia) nos lleva a la conclusión de que los responsables encuestados desconocen la naturaleza de las acciones de comunicación, siendo el origen de posteriores confusiones.

Asimismo, los gerentes y responsables de PYMES desconocen, en gran medida, el abanico de posibilidades que ofrece la comunicación, aunque reconocen la necesidad de desarrollarla a fin de lograr su expansión. Un alto porcentaje identifica comunicación con "tecnología", "información de los mercados y clientes", entre otras expresiones. No existen planes de comunicación estructurados y definidos, ni a corto ni a medio plazo (45.45\%), no ya de cara a la exportación, sino tampoco a nivel nacional. Los que afirman que cuentan con dicho plan, apenas saben explicar en qué consiste, describiéndolo de forma ambigua.

Las principales acciones de comunicación trazadas fueron: a) la asistencia a ferias, tanto nacionales como internacionales; b) edición de catálogos y folletos de productos; $c$ ) aparición de anuncios en revistas del sector; $d$ ) comunicación a través del "boca a boca"; $e$ ) degustaciones en el punto de venta, en el caso de los productos agroalimentarios; y f) cuñas de radio o anuncios en prensa, en relación con noticias concretas a nivel nacional.

Llama la atención el uso de medios (prensa y radio circunscritos al ámbito nacional) de cara a posicionar los productos en los mercados exteriores. En todo caso, estas acciones no se desarrollan en el mercado en el que se pretende actuar, ayudando poco a las empresas en el proceso de expansión hacia otros mercados exteriores.

Asimismo, se detecta una cierta actitud de conformismo con las acciones de comunicación llevadas a cabo hasta el momento. La materia comunicativa queda relegada a un segundo plano y se percibe desinterés ante la puesta en marcha de acciones de comunicación distintas a las ejecutadas rutinariamente en los últimos años.

Los gerentes de las PYMES segovianas consideran que el papel de la comunicación en el proceso de internacionalización de la empresa es "positivo" por las razones que presentaban en las respuestas del bloque de "comunicación". Enumeramos las frases textuales: "La comunicación es totalmente necesaria para dar a conocer un producto, su calidad y dar a conocer la imagen de la empresa"; "Aporta clientes y ayuda a que el consumidor comprenda la diferencia de precios, porque este busca referencias a través de la comunicación"; "Ayuda a la empresa a conocer nuevos mercados"; "Ayuda a la empresa a conocer las tendencias del mercado y el sector en el que se mueve"; "Mantiene viva a la empresa en el 
mercado y aporta imagen de seriedad a la misma"; "Es la forma de establecer relación con potenciales clientes y mantener los ya existentes"; "Ayuda a contactar directamente con proveedores y clientes"; "Mediante la comunicación se hace la presentación de avances y estudios realizados".

A la vista de los resultados obtenidos, no se potencian las herramientas de comunicación: su uso queda restringido a los cánones habituales y utilizados hasta el momento. Esta limitación (por desconocimiento de las acciones de comunicación) implica un obstáculo en el uso adecuado y eficaz de las herramientas de comunicación, de cara a la internacionalización de los productos de las empresas analizadas en esta investigación.

Debido a esta problemática, es necesario realizar otros estudios similares en el ámbito nacional e internacional, debido a la importancia que tiene la pequeña y mediana empresa para el desarrollo del tejido empresarial. No hay duda que la comunicación puede aportar decisivamente en la consecución de los objetivos empresariales.

\section{Fuentes consultadas}

Arenas, J. y García, R. (2005). “Alianzas españolas con éxito en los mercados internacionales”. Marketing \& Ventas, 71, 21-23.

Basterrechea, E. (2003). La utilización de las Tecnologías de la Información y las Comunicaciones en Segovia. Mesa redonda. Mayo 7. Segovia, España. Extraída el 30/I/2006 desde www.cajasegovia. es/observatorio/observatorio.asp

Belío, J. L. (2002). “¿Internacionalización? Sí, pero...”. MK Marketing + Ventas, 167, 9-12.

CEAC (2000). Biblioteca de gestión de la pequeña y mediana empresa (vol. 3, pp. 127-146). Barcelona: Grupo Editorial CEAC.

Boronat, D. (2003). “Internacionalizarse.com: ¿mito o realidad?”. Harvard Deusto, 55, 38-40.

Buendía, A. (2004). “Las pymes y sus historias”. Estrategias de marketing, 135, 32 y ss.

Carbonara, N. (2005). "Information and communication technology and geographical clusters: opportunities and spread". Technovation, 25(3), 213-222.

Carrillo M. V.; Castillo A. y Gómez, L. (2005). Imagen y comunicación en las PYMES. Madrid: Fragua.

Cervera Fantoni, A. L. (2004). Comunicación total. Madrid: Editorial ESIC.

“Consejo Regional de Cámaras Oficiales de Comercio e Industria de Castilla y León” (2011). Extraída el 6/IX/2011 desde http://www.camarascyl.es/cocicyl/cm

Dana, L. P. (2005). "The process of Internationalization". International Small Business Journal, 23(2), 219-221.

De Anta, A. (2005). “El futuro de las pymes”. Extraída el 26/X/2005 desde http://www.noticias.com/ 
articulo/22-05-2005/alex-anta/futuro-pymes-410d.html

Díaz, C. (2001). Internacionalización de la economía segoviana. Ciclo de ponencias. Mayo 3. Extraída el 31/V/2004 desde www.cajasegovia.es/observatorio.asp

DIRCOM (2005). "El estado de la comunicación en España”. MK Marketing + Ventas, 204, 9-11.

Edwards, T.; Delbridge, R. y Munday, M. (2005). "Understanding innovation in small and mediumsized enterprises: a process manifest”. Technovation, 25(10), 1119-1127.

“España, país de PYMES” (2006). Extraída el 12/V/2006 desde: http://www.infojobs.net/cr_recurso. cfm?id=952827206

“Especial Comercio Exterior” (2006, Febrero 18). El Adelantado de Segovia, pp. 2-6.

Etemad, H. y Wright, R. W. (2003). "Internationalization of SMEs (pymes): Toward a new paradigm". Small Business Economics, 20, 1-4.

Folgado, J. A. (2001). Internacionalización de la economía segoviana. Ciclo de ponencias. Mayo 3. Segovia, España. Extraída el 31/V/2004 desde www.cajasegovia.es/observatorio.asp

Herranz de la Casa, J. M. (2006). La comunicación y la transparencia en las organizaciones no lucrativas [Tesis]. Madrid: Departamento de Periodismo, Universidad Complutense de Madrid.

Hollenstein, H. (2005). "Determinants of international activities: Are SMEs different?". Small Business Economics, 24, 431-448.

"Las PYMES españolas a la cabeza de Europa en previsión de crecimiento" (2008). Extraída el 6/ IX/2011 desde http://www.formapyme.com/articulos/44/20/297/Innovacion-y-Empresa/Las-PYMEsespanolas-a-la-cabeza-de-Europa-en-prevision-de-crecimiento.html

“Noticias de las Cámaras de Comercio" (2005). Revista Cámaras, núms. 77-78, pp. 10-11.

Ontiveros, E. (2004). “Temor a emprender”. Empresa global, abril-2004, 21-48.

Pennings, J. M. E. y García, P. (2004). "Hedding behavior in small and medium-sized enterprises: The role of unobserved heterogeneity". Journal of Banking \& Finance, 28(5), 951-978.

Villafañe, J. (coord.) (2001). El estado de la publicidad y el corporate en España y Latinoamérica. Madrid: Pirámide. 